\title{
Electrochemical Impedance Spectroscopy (EIS) of NaCl- Saturated Sandy Soil at Sub-zero Temperatures
}

\author{
Zhiwei Chen, Pengju Han ${ }^{*}$, Bin He ${ }^{*}$, Funan Sun, Xiangling Bai, Xingyi Wang, Tiantian Guo and \\ Xiaoyuan Wang \\ College of Civil Engineering, Taiyuan University of Technology, Taiyuan, 030024, China \\ *E-mail: 13834569544@163.com, hebin@tyut.edu.com
}

Received: 5 May 2021 / Accepted: 25 June 2021 / Published: 10 August 2021

In this paper, $\mathrm{NaCl}$-saturated sandy soil at sub-zero temperatures was studied with electrochemical impedance spectroscopy (EIS). By considering the microstructure of the NaCl-saturated sandy soil, an equivalent circuit (EC) model $\left(R_{\mathrm{CCP}}\left(C_{\mathrm{IP}}\left(C_{\mathrm{DCP}} R_{\mathrm{DCP}}\right)\right)\right)$ is proposed on the basis of three kinds of conductive paths (continuous conductive paths (CCPs), discontinuous conductive paths (DCPs), and insulating paths (IPs). The correctness and suitability of the EC model are indicated by the small values of the chi-square statistic and fitting errors. The four circuit elements in this equivalent circuit model are associated with soil components, and the freezing of water to ice is the main cause of changes in their values. The fitting results obtained by this equivalent circuit model suggest that the values of the $R_{\mathrm{CCP}}$ and $R_{\mathrm{DCP}}$ increase exponentially with decreasing temperature. There is an obvious change in the trends for the values of the $C_{\mathrm{DCP}}$ and $C_{\mathrm{IP}}$ that depends on the freezing temperature. The proposed EC model can successfully explain the EIS results and help provide a better understanding of the microstructure of frozen soil. In addition, the model can be applied in other types of frozen soils and support studies of the corrosion of steel buried in soils at sub-zero temperatures, which can further broaden the application of EIS.

Keywords: Electrochemical impedance spectroscopy (EIS); Equivalent circuit (EC) model; Sandy soil; Sub-zero temperature

\section{FULL TEXT}

(C) 2021 The Authors. Published by ESG (www.electrochemsci.org). This article is an open access article distributed under the terms and conditions of the Creative Commons Attribution license (http://creativecommons.org/licenses/by/4.0/). 